\title{
Effectiveness and safety of minilaparoscopy-guided spleen biopsy: a retrospective series of 57 cases
}

\author{
Tobias Werner · Johannes Koch • Ulrike W. Denzer
}

Received: 22 May 2013/Accepted: 24 June 2013/Published online: 23 July 2013

(C) Springer Science+Business Media New York 2013

To the Editor,

We read with interest the comments from Yunqiang Cai and Xubao Liu on our paper. Proper patient selection is important when considering splenic biopsy as it is when considering biopsy of other intra-abdominal organs. To your respective comments:

(1) In patients with disseminated infections, such as tuberculosis, peritoneal dissemination from splenic biopsy is likely not relevant for the outcome of the patients, because these patients will receive systemic medical treatment of these infection. Furthermore, in patients with splenic involvement of tuberculosis, there is frequent concomitant peritoneal disseminated disease at time of diagonsis. Benign cystic lesions or hemangioma with pathagnomonic findings on imaging studies would not require biopsy.
(2) In nine of ten patients with focal lesions, these lesions were easily identified during the procedure. In patients in whom a focal lesion not identified during laparoscopy, a biopsy directed at the site identified on imaging would be advised. Multiple passess with the needle could be considered.

(3) Minilaparoscopic-guided spleen biopsy is a minimally invasive procedure performed under conscious sedation and therefore is significantly less morbid than a surgical approach, such as (partial-)splenektomie for tissue diagnosis alone. In special circumstances, such as solitary metastasis with a curative treatment option, a surgical approach should be preferred.

Disclosures Dr. Tobias Werner, Dr. Johannes Koch, and PD Dr. Ulrike W. Denzer have no conflicts of interest or financial ties to disclose.
T. Werner $(\square)$

Department of Medicine I, University Medical Center Hamburg Eppendorf, Martinistraße 52, 20246 Hamburg, Germany

e-mail: t.werner@uke.de

J. Koch

Digestive Disease Institute, Virginia Mason Medical Center,

Seattle, WA, USA

U. W. Denzer

Clinic for Interdisciplinary Endoscopy, University Clinic

Hamburg Eppendorf, Hamburg, Germany 